\title{
Gradient form and sequential effects during generalization testing in extinction
}

\author{
LAWRENCE L. CRAWFORD, KENNETH M. STEELE, and JOHN C. MALONE, JR. \\ University of Tennessee, Knoxville, Tennessee 37916
}

Four naive pigeons were given six generalization tests in extinction after periods of pretraining in which $\mathrm{S}+$ appeared with food reinforcement and $\mathrm{S}-$ appeared in extinction. An analysis of sequential effects among presentations of test stimuli showed that the overall gradient was influenced differently by stimuli at the extremes of the continuum of test stimuli and by $\mathrm{S}+$ and adjacent stimuli. Gradients consisting of responding in each stimulus when it was preceded by an extreme stimulus tended to peak at $\mathrm{S}+$, while gradients produced when each stimulus was preceded by S+ or an adjacent stimulus tended to show a peak shift. This was true whether the overall gradient showed a peak shift or not. Two naive subjects were added and four additional tests were given after pretraining in which unequal frequencies of reinforcement accompanied both S+ and S-. Results of all 10 tests show that sequential effects occur during generalization testing in extinction and that these "local dimensional effects" are unlike local contrast. These stimulus-specific sequential effects may greatly influence overall gradient form.

Most recent researchers have viewed generalization gradients obtained during extinction testing as probes which reveal dispositions to respond dependent wholly on conditions of pretraining (e.g., Friedman \& Guttman, 1965; Mackintosh, 1974; Thomas, 1974) rather than on the specific conditions obtaining during a generalization test. In large part, this attitude seems justified, because a great deal of research shows that many specific conditions of testing do not greatly affect the form of the gradients obtained. For example, Hiss and Thomas (1963) reported no consistent differences in gradient form between birds exposed to the entire test series when compared to the combined gradients of birds individually exposed to only one of the test stimuli during extinction. Friedman (1963) showed that the form of generalization gradients depended upon the absolute (e.g., physical) values of test stimuli rather than their relative spacing within the test series. Following discriminative pretraining, Stevenson (1966) showed very convincingly that the same gradient forms originally reported by Hanson (1959) appeared even when only half the range of stimulus values used by Hanson was presented during the test.

There is reason, however, to believe that sequential effects during (intradimensional) discriminative pretraining and/or generalization testing may be important in influencing gradient form. The evidence for this comes from investigations of the peak shift.

This research was supported by Grant MH-29774 from the National Institute of Mental Health, USPHS, and by a University of Tennessee Faculty Research Grant to the third author. Reprints may be obtained from any of the three authors, Department of Psychology, University of Tennessee, Knoxville, Tennessee 37916.
Positive peak shift, first described by Hanson (1959), refers to the displacement of the peak of the gradient from the stimulus presented with reinforcement during pretraining $(\mathrm{S}+)$ to a new stimulus on the test continuum in a direction away from the training stimulus with the lower frequency of reinforcement (S-). Certain aspects of Hanson's peak shift, such as the increased height and sharpening of the gradient form, were evaluated by reference to a control group trained with $\mathrm{S}+$ alone. However, the most visually distinct feature, the shifted peak of responding to a stimulus not previously encountered during training, was readily apparent from an examination of the discriminative training group alone.

Friedman and Guttman (1965), in a series of experiments, showed that the shift occurred only after successive discrimination training (i.e., $\mathrm{S}+$ and $\mathrm{S}-$ successively present, as in a multiple schedule). Prior training with $\mathrm{S}+$ alone and a subsequent extinction session with $\mathrm{S}-$ alone produced no shift, but only lowered the overall gradient obtained in an extinction test. Furthermore, Ellis (1970) showed that pretraining must include at least one $\mathrm{S}-$ to $\mathrm{S}+$ transition per session if the peak shift is to appear during a subsequent generalization test. The dependence of the peak shift on the stimulus sequence during pretraining suggests that sequential effects may be important in determining gradient form.

Similarly, the stimulus sequence during testing may influence gradient form. Hanson (1956), in the doctoral dissertation which preceded his (1959) published report, mentioned that sequential effects occurred during testing, but no systematic record was made of them. Later, Donahoe, McCroskery, and Richardson (1970) described sequential effects during 
extinction testing and interpreted them as similar to Pavlovian induction effects. Malone (1976) has argued that local contrast (Malone \& Staddon, 1973; Nevin \& Shettleworth, 1966), a sequential effect, is directly analogous to Pavlovian induction. Local contrast appears as large increases and decreases in response rate during one stimulus presentation as a function of the immediately preceding stimulus. When the preceding stimulus is associated with a lower frequency of reinforcement or a lower average response rate, responding during the subsequent stimulus is augmented (positive local contrast). When the preceding stimulus is associated with a higher frequency of reinforcement or a higher average response rate, responding in the subsequent stimulus is suppressed (negative local contrast). Local contrast may fade with continued training (Nevin \& Shettleworth, 1966) but clearly influences response rates while it lasts (Malone, 1976). Most interestingly, local contrast could contribute toward what will be asymptotic rates of response and thus influence behavioral contrast (e.g., Mackintosh, 1974).

More recent data suggest even more strongly that local (sequential) effects during pretraining and testing may be important in determining gradient form. Malone and Rowe (in press) found that generalization gradient form during maintained generalization training, in which reinforcement and nonreinforcement were maintained in stimuli along a continuum over many sessions of training, was strongly affected by local/sequential effects. The final form of the gradients, as shown in average response rates, was anticipated many sessions earlier in gradients comprised of response rates in each stimulus when preceded by specific stimuli, in this case the extremes of the continuum. These sequential effects could well have played a part in determining the final form of the gradients.

The experiment below examined the influence of sequential effects among stimulus presentations during generalization testing in extinction. In the first four tests, generalization testing was preceded by discrimination training in which $\mathrm{S}+$ was accompanied by variable-interval (VI) reinforcement and $S-$ appeared in extinction. The next two tests were preceded by training with an $\mathrm{S}$ - more similar to the $\mathrm{S}+$. The last four tests, which included two new naive subjects, were all preceded by training in which $S+$ and $S$ - were accompanied by two different VI reinforcement schedules. The typical S+ only control group was not used in this experiment for two reasons. The lesser reason was that such a control group has not always been considered a necessity when the issue is the existence of the shifted peak per se (e.g., Friedman \& Guttman, 1965; Terrace, 1968). More importantly, the aim of this study was the examination of the influence of sequential effects during generalization testing on overall gradient form. While the procedure was similar to that of previous studies designed to demonstrate peak shift, such a demonstration was the main object here. Nonetheless, the elimination of the control group used by Hanson and by others does not prevent some comparisons with his data, such as the demonstration of the increased area and the sharpening of the gradient for the discriminative pretraining subjects.

Of course, the procedure here was already different from that of most previous studies in that the data were analyzed for individual subjects rather than averaged over a number of subjects. Furthermore, unlike typical previous studies, the subjects here were repeatedly tested following interpolated periods of discrimination training.

\section{METHOD}

\section{Subjects}

Six adult, experimentally naive White Carneaux pigeons were reduced to $80 \%$ of their free-feeding weights and remained at these weights for the duration of the experiment.

\begin{abstract}
Apparatus
The experimental chamber was a $28 \times 26 \times 31 \mathrm{~cm}$ steel and Plexiglas box that was entirely enclosed in a larger soundproofed box. A Grason-Stadler stimulus projector mounted behind the transparent response key provided .3-cm-wide black line-tilt stimuli in 15-deg increments on a white background. The key was centered over the $5.2 \times 5.7 \mathrm{~cm}$ magazine aperture. During reinforcement ( $3 \mathrm{sec}$ access to mixed grain), the stimulus on the key was extinguished, but the 6-W houselight remained on throughout the session. The soundproofed box and a large ventilation fan, as well as white noise, masked outside noises. All programming and recording equipment was located in an adjoining room.
\end{abstract}

\section{Procedure}

Keypecking was established through an autoshaping procedure (Brown \& Jenkins, 1968) with a 30-deg (from horizontal) line orientation projected on the key. Birds $70,77,78$, and 79 then began pretraining for the first set of generalization tests. During each daily session, $S+(30 \mathrm{deg})$ and $S-(60 \mathrm{deg})$ appeared for alternating $30-\mathrm{sec}$ presentations. Each training session lasted approximately $30 \mathrm{~min}$. Responding in $\mathbf{3 0} \mathrm{deg}$ was reinforced according to a VI 30 -sec schedule, while $60 \mathrm{deg}$ appeared in extinction. After 14 sessions, the first generalization test was given. During the test presentations, seven line-orientation stimuli [0 (horizontal), $15,30,45,60,75$, and $90 \mathrm{deg}$ (vertical)] appeared for successive 15 -sec periods in extinction with no warm-up. The presentation order of test stimuli was arranged so that each stimulus was preceded by itself and each other stimulus once within each of three rounds of 49 presentations per round. The order of stimulus presentations was the same for all subjects during any given test. Responding during each stimulus presentation was recorded for analysis of sequential effects. After Generalization Test 3, a 2-sec time-out was inserted between all stimulus presentations in both training and test sessions.

After intervening periods of discrimination pretraining, three further extinction tests were given. Generalization Test 2 was preceded by 12 sessions of pretraining, Test 3 by 8 sessions, and Test 4 by 14 sessions. After the first four tests, $S-$ was changed from 60 to $45 \mathrm{deg}$ and two more tests (5 and 6) were given. Test 5 was preceded by 14 sessions of pretraining and Test 6 was preceded by 10 sessions of pretraining.

For the last four tests ( 7 through 10 ), two additional naive birds, 71 and 80 , were added, following a period of autoshaping similar to that given the original four birds. During pretraining for Tests 7 
and 8 , both $S+(30 \mathrm{deg})$ and $S-(60 \mathrm{deg})$ were presented with VI reinforcement: $S+$ appeared with VI $30-\mathrm{sec}$ and $S-$ with VI 2 -min reinforcement. Both Tests 7 and 8 were preceded by 18 sessions of pretraining. The final two tests $(9$ and 10$)$ were preceded by discrimination training similar to that preceding Tests 7 and 8 , but $\mathrm{S}$ - was $45 \mathrm{deg}$, as in Tests 5 and 6 . Test 9 was preceded by 22 sessions of pretraining, and Test 10 was preceded by 23 sessions of pretraining.

\section{RESULTS}

The presentations of test stimuli were only $15 \mathrm{sec}$ long so that all 147 presentations (three rounds of 49 each) could appear during a relatively brief session of slightly less than $37 \mathrm{~min}$. In this way, it was hoped that substantial responding would continue through the test session in extinction. But after the first few tests, responding during the third, and often the second, rounds became so low as to be negligible for some birds. Consequently, only the data from the first round of each test will be discussed. While there were often changes in gradient form from the first round to the second and third, the same changes occurred between tests. Particularly during the first four tests, changes in gradient form with successive rounds within a test appeared to be very similar to the changes in gradient form in Round 1 across successive tests. Thus, little was lost in discounting second and third round data. Almost all of the data below derive from individual birds rather than from group averages, and, considering this, the data were quite orderly. Only in the last figure were the data converted to relative response rates and averaged over subjects, and this was done to allow one important comparison summarizing the overall effect of sequential effects on gradient form.

\section{Tests 1-4: After Pretraining with $30 \mathrm{deg}(\mathrm{S}+, \mathrm{VI})$ and $60 \operatorname{deg}(\mathrm{S}-$, EXT)}

Figure 1 shows the overall influence of sequential effects on overall gradient form for each bird during each test. Dashed lines show overall gradients (each test stimulus preceded by itself and each other), which is thus the sum of all sequential effects, while solid lines show response rates in each stimulus when preceded by itself, thus minimizing the sequential effects produced by other stimuli. The rationale for using stimulus-preceded-by-itself as a baseline against which to assess sequential effects was first proposed by Jenkins (1965) and was later used by Malone (1976) and Malone and Staddon (1973). This figure was intended only to give a gross picture of the influence of sequential effects on overall gradient forms. In this and all figures to follow, the $\mathrm{S}+$ used in pretraining ( $30 \mathrm{deg})$ is indicated by a filled circle.

All birds showed a peak shift in the first test, though Bird 79 showed two peaks (at 0 and $30 \mathrm{deg}$ ) in its overall gradient (dashed line). By Test 2, the shift was absent in the overall gradient for all but Bird 70 ,

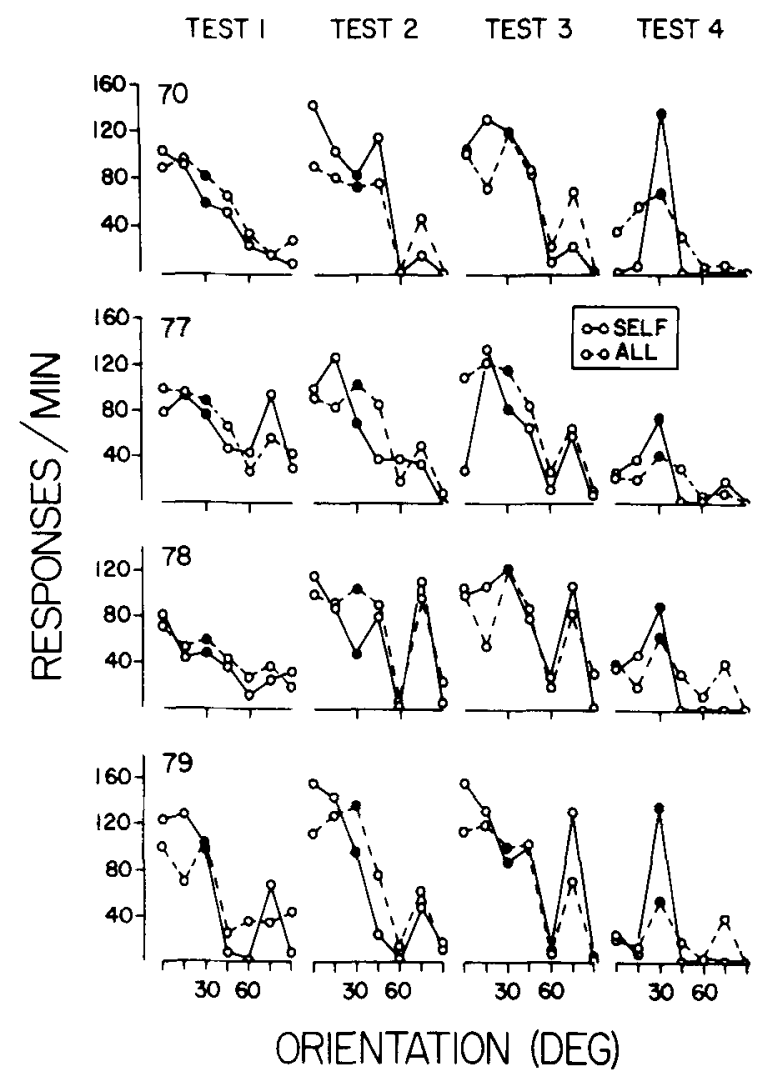

Figure 1. Gradients for each bird during the first four extinction tests, in which $S+$ was 30 deg and $S-$ was 60 deg. Points connected with dashed lines show overall gradients during the first round of test stimuli. Solid lines show gradients resulting from those instances in which a stimulus was preceded by itself. $S+$ is indicated by filled circles.

while each bird still showed a shift in the stimuluspreceded-by-itself gradients. In the third test, the results were mixed, with a peak at $\mathrm{S}+$ in both gradients for Bird 78 and a shift in both for Birds 77 and 79. Bird 70 showed what the others had in the preceding test, a shift in the stimulus-preceded-by-itself gradient, but none in the overall gradient.

The fourth test resulted in peaks at $\mathrm{S}+$ in both sets of gradients for all birds, though the peaks were less pronounced in the overall gradients. Overall, the peak shift was lost most rapidly in the overall gradients during the first three tests, suggesting that sequential effects act to abolish the peak shift.

Of course, the differences in the pairs of gradients in Figure 1 could simply depend upon the number of data points comprising them, rather than upon the action of sequential effects. Therefore, a careful examination was made of sequential effects produced by all stimuli during each test. Gradients were plotted which showed response rates in each stimulus when preceded by each stimulus; i.e., gradients for each bird during each round when each stimulus was preceded by 0 deg, other gradients when each stimulus was preceded by $15 \mathrm{deg}$, and so on. The main influence of stimulus-specific sequential effects on gradi- 

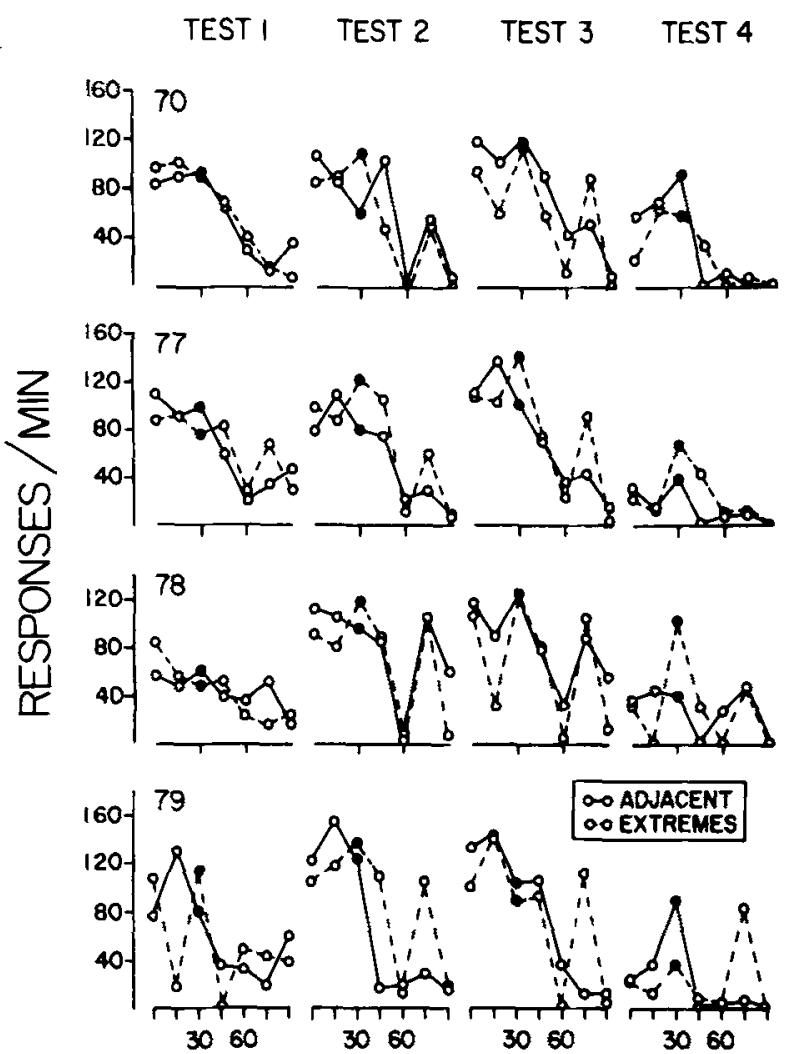

\section{ORIENTATION (DEG)}

Figure 2. Gradients for each bird during the first four extinction tests. Points connected by dashed lines show gradients resulting from instances in which each stimulus was preceded by one of the extreme stimuli $(0,75$, or 90 deg). Solid lines show gradients including oaly those instances in which each stimulus was preceded by $S+$ or an adjacent stimulus (i.e., 15, 30, or $45 \mathrm{deg}$ ). $S+$ is indicated by filled circles.

ent form is shown in Figure 2, which shows gradients produced when each stimulus was preceded by $\mathrm{S}+$ or an adjacent stimulus (i.e., 15, 30, or $45 \mathrm{deg}$ ) or by a stimulus representing one of the extreme values of the continuum $(0,75$, or $90 \mathrm{deg})$. The division between extreme and other stimuli was somewhat arbitrary; had $30 \mathrm{deg}$ been left out of the "adjacent" group and 75 deg been left out of the "extremes" group, the data would have appeared virtually the same.

As in Figure 1, gradients during the first round of stimulus presentations are shown for each bird for each test. Solid lines show gradients when each stimulus was preceded by $\mathrm{S}+$ or an adjacent stimulus, while dashed lines show gradients when each stimulus was preceded by one of the extremes. A peak shift appeared in the "adjacent" gradients in 10 of 16 cases and in the "extremes" gradients in only 5 of 16 cases. The tendency of the extreme test stimuli to abolish the peak shift and place the peak at the pretraining $\mathbf{S}+$ was apparent across tests and across the three rounds of each test. While the latter data are not included here, the figures which follow provide more than enough illustrations of the effects of extreme vs. $\mathrm{S}+$ and adjacent stimuli on the peak shift.

\section{Tests 5 and 6: After Pretraining with 30 deg} (S+, VI) and $45 \mathrm{deg}(\mathrm{S}-, \mathrm{EXT})$

Figure 3 shows gradients for each bird averaged over the fifth and sixth extinction tests, where $\mathrm{S}+$ was again $30 \mathrm{deg}$, but $\mathrm{S}-$ was $45 \mathrm{deg}$. $\mathrm{S}+$ is again indicated by a filled circle. The gradients on the left were those produced when each stimulus was preceded by $\mathrm{S}+$ or an adjacent stimulus, the middle column of gradients was produced when each stimulus was preceded by one of the extreme test stimuli, and the gradients on the right represent the overall gradients.

\section{ADJACENT EXTREMES OVERALL}
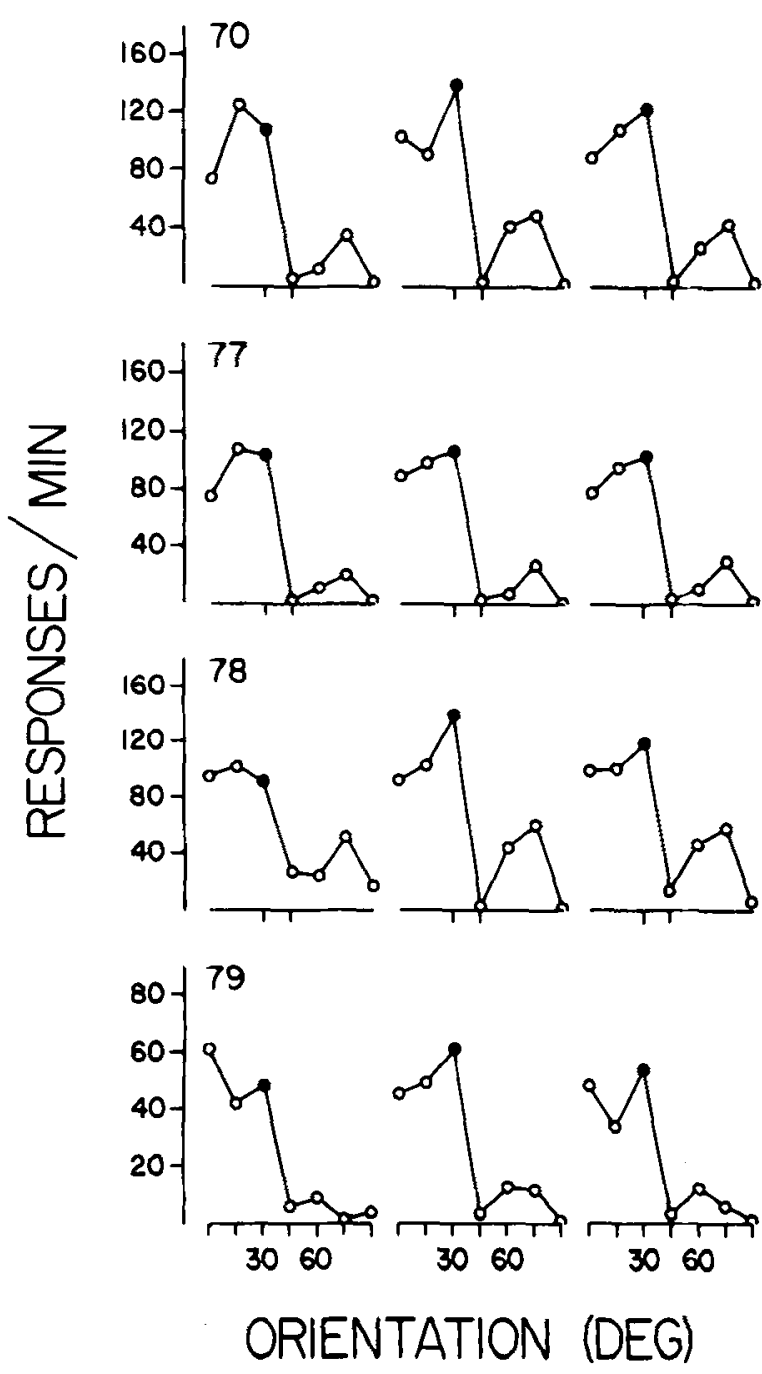

Figure 3. Data for individual birds averaged over Tests 5 and 6 , in which S+ was 30 deg and S- was 45 deg. Overall gradients are shown on the right, and gradients produced when the preceding stimulus was one of the extremes $(0,75$, or 90 deg) appear in the center. Gradients on the left were produced when only those instances in which the preceding stimulus was $S+$ or an adjacent stimulus were included. See text for details. 
The overall gradients showed a peak at $\mathrm{S}+$ in all cases, as was true for the gradients produced by the extreme stimuli. But when $\mathrm{S}+$ or an adjacent stimulus preceded each stimulus, a peak shift occurred in all cases. The difference in gradient forms was not confined solely to the end of the continuum in which the shift appeared; the form of the right half of the gradient for the "extremes" gradient conformed more closely to the right half of the overall gradient than did the "adjacent"' gradient.

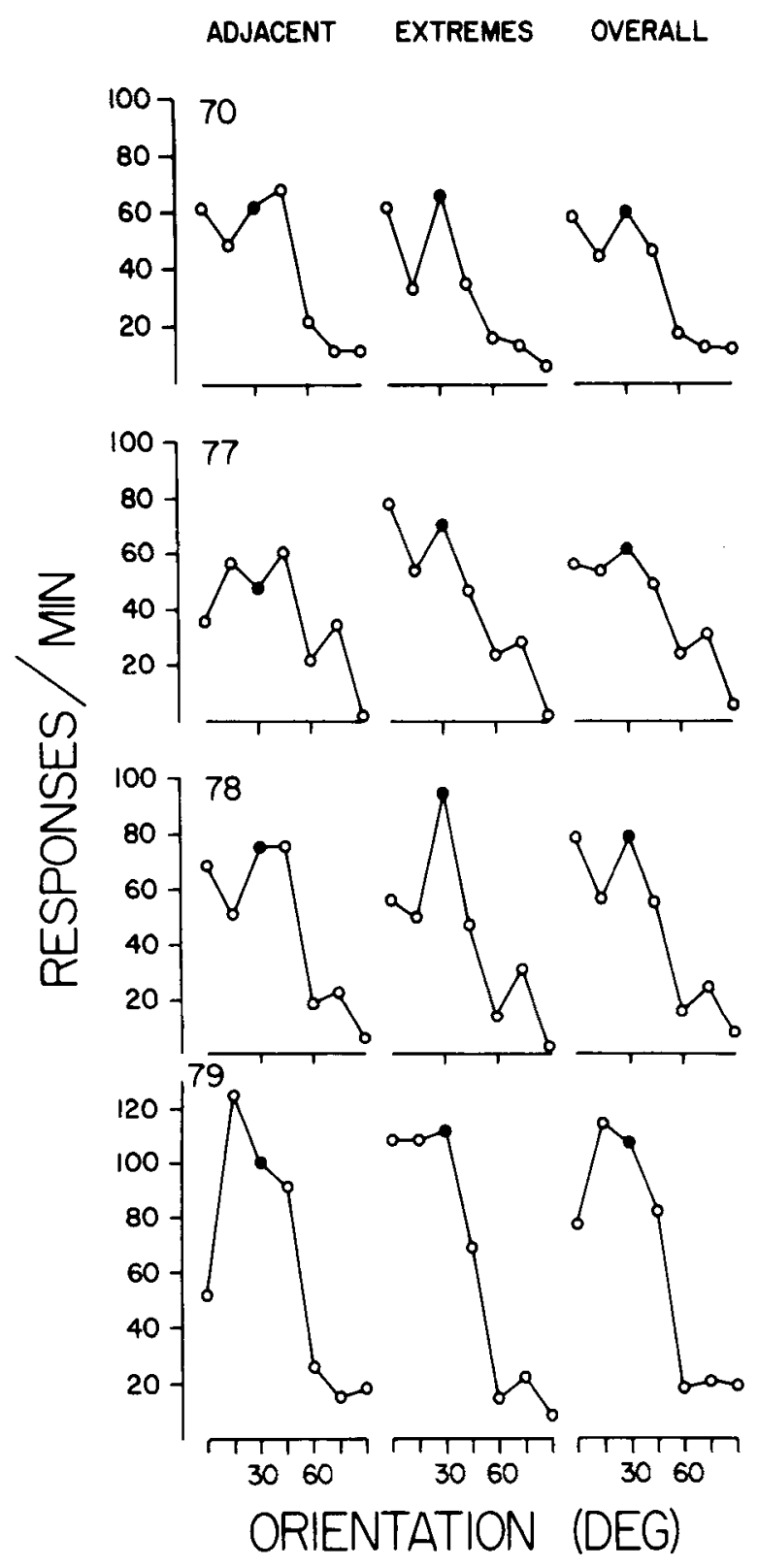

Figure 4. Data for individual birds averaged over Tests 7 and 8 , in which $S+(30 \mathrm{deg})$ and $S-(60 \mathrm{deg})$ were both accompanied by VI reinforcement during pretraining. Overall gradients appear on the right, while the center and left columns show gradients produced when each stimulus was preceded by one of the extreme stimuli or by $\mathbf{S}+$ and adjacent stimuli.

\section{ADJACENT EXTREMES OVERALL}
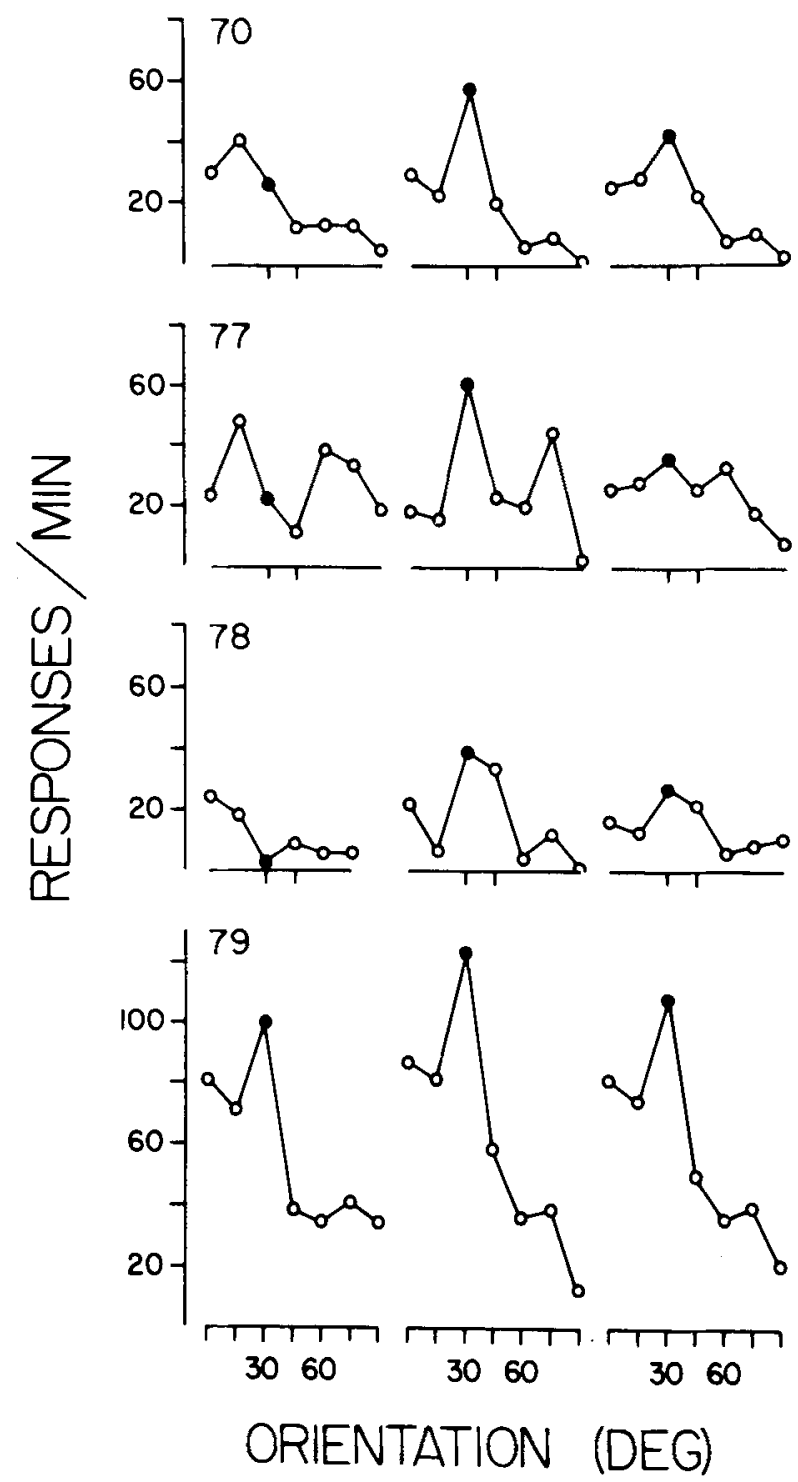

Figure 5. Data for individual birds averaged over Tests 9 and 10 , in which $S+(30 \mathrm{deg})$ and $S-(45 \mathrm{deg})$ were both accompanied by VI reinforcement during pretraining. Overall gradients appear on the right, while the center and left columns show gradients produced when each stimulus was preceded by one of the extreme stimuli or by $\mathbf{S}+$ and adjacent stimuli.

\section{Tests 7 and 8: After Pretraining with $30 \mathrm{deg}$ $(\mathrm{S}+, \mathrm{VI})$ and $60 \mathrm{deg}(\mathrm{S}-, \mathrm{VI})$}

Figure 4 shows similar data for the next two tests, in which both stimuli appeared with VI reinforcement during pretraining (VI 30-sec and VI 2-min). Overall gradients showed a shift in one case (Bird 79), peaks at both $\mathrm{S}+$ and $0 \mathrm{deg}$ (Birds 70 and 78), and one peak at $\mathrm{S}+$ (Bird 77). Gradients produced by the extreme stimuli showed peaks at $\mathrm{S}+$ in three of four cases, though responding was also high in $0 \mathrm{deg}$ for Bird 79. The gradients produced by $\mathrm{S}+$ and adjacent stimuli (left column) in no case peaked at $\mathrm{S}+$. As in the previous tests, the gradients produced by 
the extreme stimuli preceding each other stimulus tended to peak at $\mathrm{S}+$, though it was clear that the VI/VI pretraining led to less clear gradients than was the case for the earlier tests. This was particularly true for the "adjacent" gradients.

\section{Tests 9 and 10: After Pretraining with 30 deg} (S+, VI) and $45 \mathrm{deg}(\mathrm{S}-$, VI)

Figure 5 shows the results of the last pair of extinction tests, averaged for each bird and arranged as were the preceding two figures. Again, pretraining involved the same reinforcement schedules as used prior to Tests 7 and 8 , but S- was $45 \mathrm{deg}$, an orientation more similar to $S+$. In all cases, the overall gradients showed a peak at $\mathrm{S}+$, though the gradients for Birds 77 and 78 were quite flat. The reason for this is easy to see when one considers the "adjacent" gradients; those two birds showed particularly irregular gradient forms, with nothing like a peak at S+. The slight peak in the overall gradients is due to the effects of the extreme stimuli (center column).

Bird 70 showed a peak shift in the gradient produced by $S+$ and adjacent stimuli, but a sharp peak at $\mathrm{S}+$ in the "extremes" gradient. Bird 79 showed a clear peak at $\mathrm{S}+$ in all three gradients.

\section{Tests 7-10: Added Naive Birds}

The data for Birds 71 and 80, the two naive birds added before Test 7, appear separately in Figure 6, which shows the gradients for each bird averaged over Tests 7 and 8 (60 deg as $S-)$ and Tests 9 and 10 (45 deg as $S_{-}-$). Bird 71 showed peaks at $S+$ in all three gradients in Tests 7 and 8 . In Tests 9 and 10, a peak shift appeared in the overall gradient and in the "adjacent" gradient, if that can be called a gradient. Yet, in the gradient produced by the extreme stimuli, the peak appeared (however marginally) at $\mathrm{S}+$.

Bird 80 showed very irregular gradients in Tests 7 and 8, though the closest approximation to a peak at $\mathrm{S}+$ appeared in the "extremes" gradient. During Tests 9 and 10, clear peaks appeared at $S+$ in all three gradients, though the gradient produced by the extreme stimuli was the sharpest.

The data of the two added birds, in agreement with the previously described data, showed that gradients produced by the aftereffects of the extreme stimuli were those that most clearly showed a peak at $\mathrm{S}+$ rather than a peak shift or an irregular gradient. In general, however, these results were less clear than those from the first four tests for the other birds, where VI and extinction had prevailed previously during pretraining.

\section{Overall Results: Sequential Effects and Gradient Form}

These results show that sequential interactions among test stimuli do have an influence on overall gradient form during a generalization test in extinc-

\section{AOJACENT EXTREMES OVERALL}
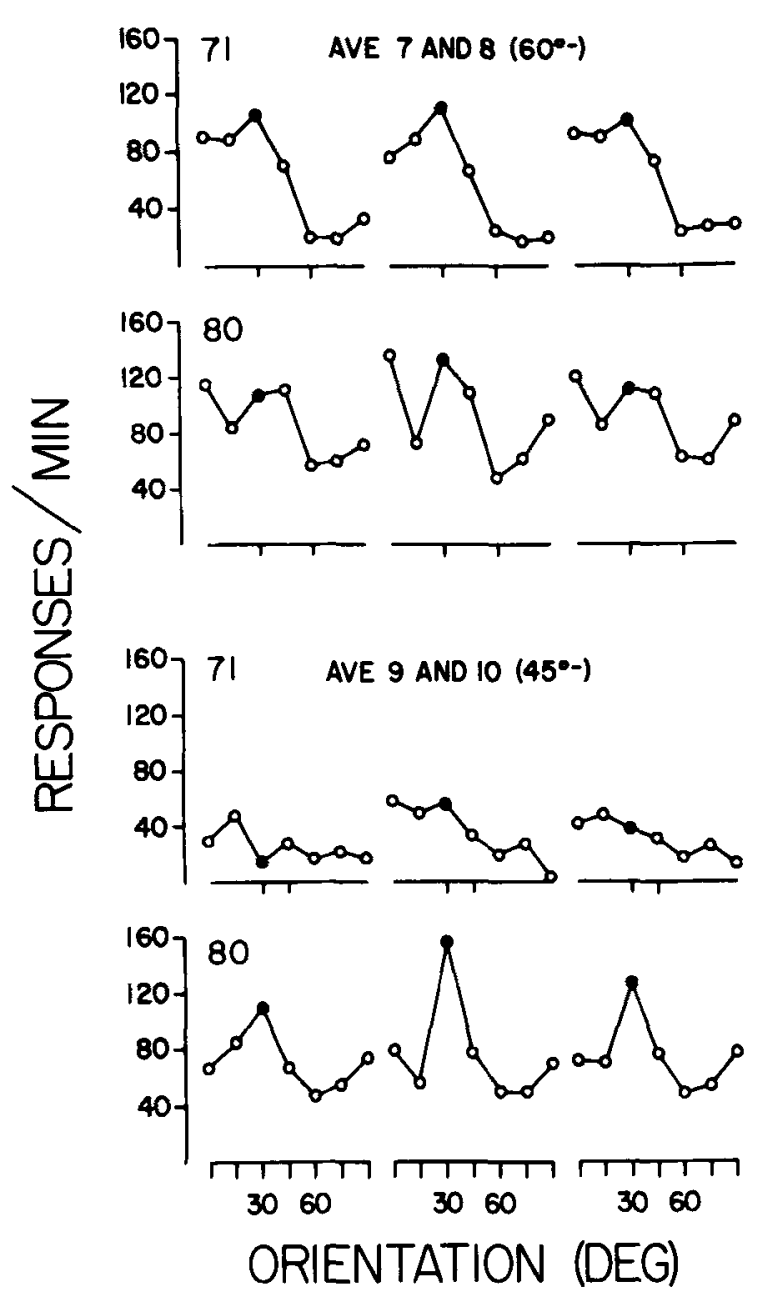

Figure 6. Data for Birds 71 and 80 avermged over Tests 7 and 8 and over Tests 9 and 10 . Both $S+$ and $S-$ were accompanied by VI reinforcement during pretraining in both cases. $S+$ is indicated by filled circles, and gradients are shown when each stimulus was preceded by the extreme stimuli or by $S+$ and adjacent stimuli. Gradients on the right are the overall gradients.

tion. Figure 7 summarizes the main effects shown in the 10 extinction tests. Since data were averaged across animals, relative response rates were used so that any differences in absolute response levels among subjects would not distort the average. The figure shows gradients produced when the stimuli preceding each stimulus were extremes (dashed lines) or $\mathrm{S}+$ and the two adjacent stimuli (solid lines). The left half of the figure shows those tests in which $60 \mathrm{deg}$ was S- and the right half those tests in which $45 \mathrm{deg}$ was $\mathrm{S}-$. The upper half of the figure shows gradients when pretraining in $\mathrm{S}+$ and $\mathrm{S}-$ was accompanied by VI reinforcement and extinction, while the lower half shows those tests in which pretraining included VI reinforcement in both $\mathrm{S}+$ and $\mathrm{S}-$. The left half of each of the two columns represents cases in which the overall gradient for the birds involved showed a peak shift, while 


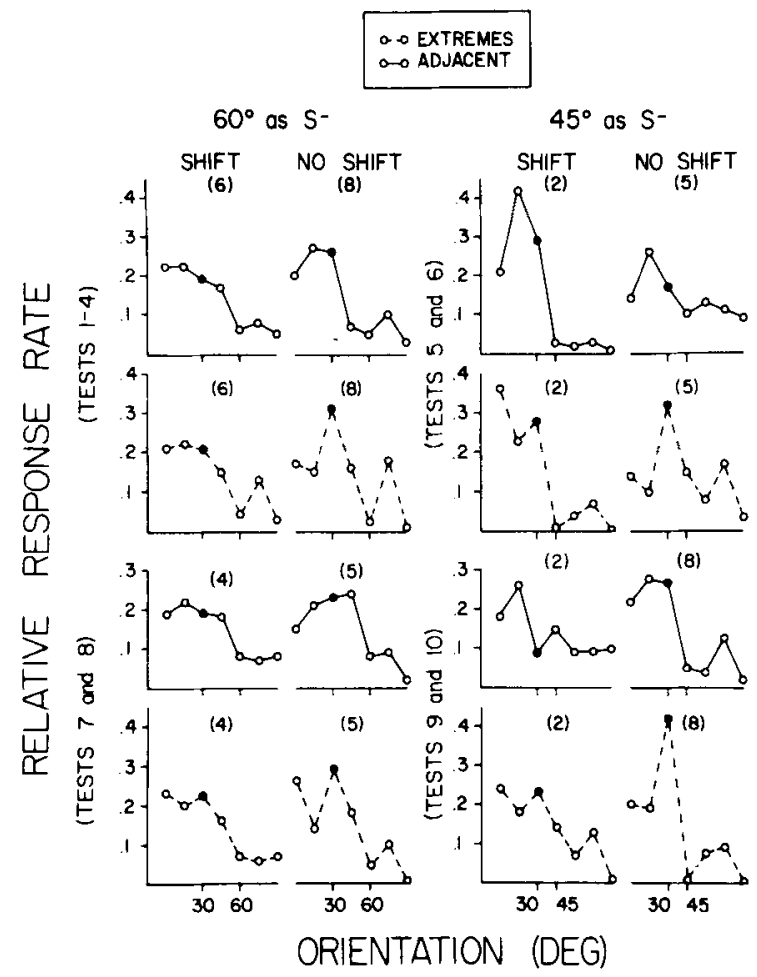

Figure 7. Averaged gradients during each condition arranged according to whether a peak shift was clearly present and whether the stimuli preceding each test stimulus were one of the extremes or $\mathrm{S}+$ and adjacent stimuli. See text for details.

the right half of each column shows cases in which the overall gradient showed a peak at $\mathrm{S}+$. The numbers in parentheses refer to the number of cases that contributed to the averaged gradient. The eight cases in which approximately equal peaks occurred at $\mathrm{S}+$ and at 0 deg were not included; only cases showing a clear peak at $\mathrm{S}+$ or a clear shift were used.

For example, consider the upper left quadrant of the figure, which shows the results for Tests 1 through 4 , in which $60 \mathrm{deg}$ was $\mathrm{S}-$ and pretraining was VI/EXT. That gradient shows that in the six cases in which there was a peak shift in the overall gradient, there was also a shift in the gradient produced when each stimulus was preceded by $\mathrm{S}+$ or an adjacent stimulus. The gradient immediately below that one shows that there was also a shift in the gradients produced by the extreme stimuli (dashed lines). The right pair of gradients in this quadrant shows that, in the eight cases in which there was no shift in the overall gradient, there was a shift in the "adjacent" gradient but a sharp peak at $\mathrm{S}+$ in the extreme gradient.

The same result appears for each condition (i.e., the averaged results of the subsequent pairs of tests). Where there was a shift in the overall gradients (the SHIFT columns), there was a shift in the gradients produced by the extreme stimuli and by the $\mathrm{S}+$ and adjacent stimuli. Where there was no shift in the overall gradients, gradients produced when each stimulus was preceded by $\mathrm{S}+$ or an adjacent stimulus showed a clear peak shift in three cases and a peak at $45 \mathrm{deg}$ in one case. For gradients produced when every stimulus was preceded by one of the extreme stimuli, the peak was clearly at $\mathrm{S}+$ in every case.

\section{DISCUSSION}

These data clearly show that sequential interactions among test stimuli can influence gradient form during generalization testing in extinction. Gradients produced when each stimulus was preceded by one of the extremes of the continuum tended to peak at the $\mathrm{S}+$ used in pretraining, while gradients produced when each stimulus was preceded by $\mathrm{S}+$ or an adjacent stimulus tended to show a peak shift, whether or not there was a shift in the overall gradient. This suggests that the form of the overall gradient could be manipulated to some degree by using different presentation sequences of test stimuli. One could probably imagine reasons, for example, for arranging a test sequence in which $\mathrm{S}+$ preceded each test stimulus. The outcome would be gradients much more likely to show a peak shift than would gradients resulting from a sequence in which, for example, each stimulus was preceded equally often by each other stimulus or was preceded only by one of the extreme values of the stimulus continuum.

Hinson, Malone, and Rowe (in press) and Malone and Rowe (in press) found similar stimulus-specific interactions using the same stimulus continuum, but with maintained generalization procedures. Hinson et al. found that local interactions among presentations of their stimuli had a great influence on the form of the maintained gradients, particularly during the early stages of training. Reinforcement was provided only for responding to the extreme two stimulus values on a continuum of line orientations ranging from vertical to horizontal, while five other orientations appeared in extinction. Between the second and third weeks of training, the form of the overall gradient was clearly dependent on which stimulus preceded the other stimuli. For example, gradients calculated by taking instances in which $45 \mathrm{deg}$ (the center of the continuum) preceded each stimulus were smooth $U$ shapes, while gradients resulting when 0 deg preceded each stimulus were $W$ forms, with the lowest response rates in extinction stimuli near 0 deg. The sum of all of the individual gradients produced by each stimulus and its aftereffects (i.e., the overall gradient) was a $\mathrm{W}$ form for each bird. Later, the individual effects produced by each stimulus disappeared, and by the fourth week all gradients showed a $\mathrm{W}$ form, regardless of the preceding stimulus.

Malone and Rowe (in press) showed similar effects in other situations and suggested that local contrast effects might pass through two stages. The first stage, which we term a local dimensional effect, occurs as a discrimination begins to form and is stimulus spe- 
cific. Local aftereffects of a given stimulus on other successively presented stimuli depend upon the specific stimulus value, as well as upon conditions of reinforcement. In the data presented here, these are the sorts of effects which were found. The second stage is what has been called local contrast (e.g., Malone, 1976; Malone \& Staddon, 1973) and is dependent only upon conditions of reinforcement or, more generally, on average response rates in each stimulus. In local contrast, two stimuli in which average response rates (over a session or more) are the same will produce, for example, suppressed responding (negative local contrast) in a subsequent stimulus when the average response rate in that stimulus is lower. This happens independently of the position of the three stimuli on a continuum.

The effects here were not local contrast as thus defined, but local dimensional effects of the stimulusspecific "first stage." Whether the second stage (local contrast) ever occurs during generalization testing in extinction is doubtful, since the transient nature of such a test does not allow the more stable differences in average response rates which seem necessary to produce local contrast.

The importance of the extreme stimuli in placing the gradient peak at $S+$ and the influence of $S+$ and adjacent stimuli in displacing the peak from $S+$ seem to beg for an explanation in terms of adaptation-level theory (e.g., Thomas, 1974). We have surveyed the literature and can find no way in which the concepts of AL theory are applicable here. Similarly, we have been unable to find much help from the literature on anchor effects and their influence on perceptual judgments, though our search has gone back to the turn of the century.

Nonetheless, the data here show that sequential effects do influence gradient form during generalization testing in extinction and that, with the stimulus continuum used here, the extreme stimuli produce effects on overall responding that are very different from those produced by $\mathrm{S}+$ and adjacent stimuli.

It could be concluded that some procedure should be used which eliminates sequential interactions during generalization testing in extinction and elsewhere that they may occur. For example, time-out periods of sufficient duration to eliminate such effects could be inserted between presentations of test stimuli. Mackintosh, Little, and Lord (1972) reported that 60-sec time-outs eliminated transient (local) contrast effects during discrimination training, while 10 -sec time-outs were ineffective. But we have recently found (unpublished data) that stimulus-specific sequential effects, similar to those described above, easily traversed 30 -sec and even 60 -sec time-out periods.

We feel that sequential effects are present (and influential) in a far wider range of settings than has been suspected and that procedures designed to eliminate them often only substitute new kinds of sequential interactions. Further investigation of the effects characterizing the process of discrimination and generalization, including sequential effects, may help us to better understand what it is that our models are to explain. An exclusive concentration on the outcomes of training procedures is probably not sufficient.

\section{REFERENCES}

Brown, P. L., \& Jenkins, H. M. Auto-shaping of the pigeon's key-peck. Journal of the Experimental Analysis of Behavior, 1968, 11, 1-8.

Donahoe, J. W., McCroskery, J. H., \& Richardson, W. K. Effects of context on the postdiscrimination gradient of stim. ulus generalization. Journal of Experimental Psychology, 1970, 84, 58-83.

ElLis, W. R., III. Role of stimulus sequences in stimulus discrimination and stimulus generalization. Journal of Experimental Psychology, 1970, 83, 155-163.

Friedman, H. Wave-length generalization as a function of spacing of test stimuli. Journal of Experimental Psychology, 1963, 65, 334-338.

Friedman, H., \& Guttman, N. A further analysis of effects of discrimination training on stimulus generalization gradients. In D. I. Mostofsky (Ed.), Stimulus generalization. Stanford: Stanford University Press, 1965.

Hanson, H. M. Effects of discrimination training on stimulus generalization. Unpublished doctoral dissertation, Duke University, 1956.

Hanson, H. M. Effects of discrimination training on stimulus generalization. Journal of Experimental Psychology, 1959, 58, 321-334.

Hinson, J. M., Malone, J. C., JR., \& Rowe, D. W. Contrast and generalization. Journal of the Experimental Analysis of Behavior, in press.

Hiss, R. H., \& Thомаs, D. R. Stimulus generalization as a function of testing procedure and response measure. Journal of Experimental Psychology, 1963, 65, 587-592.

JENKins, H. M. Measurement of stimulus control during discriminative operant conditioning. Psychological Bulletin, 1965, 64, 365-376.

Mackintosh, N. J. The psychology of animal learning. New York: Academic Press, 1974.

Mackintosh, N. J., Little, L., \& LoRd, J. Some determinants of behavioral contrast in pigeons and rats. Learning and Motivation, 1972, 3, 148-161.

Malone, J. C., JR. Local contrast and Pavlovian induction. Journal of the Experimental Analysis of Behavior, 1976, 26, 425-440.

Malone, J. C., JR., \& Rowe, D. W. Two types of local contrast and their relation to dimensional contrast. In $\mathrm{M}$. L. Commons \& J. A. Nevin (Eds.), Quantitative studies of operant behavior: Discriminative properties of reinforcement schedules. Elmsford, N.Y: Pergamon, in press.

Malone, J. C., Jr., \& Staddon, J. E. R. Contrast effects in maintained generalization gradients. Journal of the Experimental Analysis of Behavior, 1973, 19, 167-179.

Nevin, J. A., \& Shettheworth, S. J. An analysis of contrast effects in multiple schedules. Journal of the Experimental Analysis of Behavior, 1966, 9, 305-315.

Stevenson, J. G. Stimulus generalization: The ordering and spacing of test stimuli. Journal of the Experimental Analysis of Behavior, 1966, 9, 457-468.

Terrace, H. S. Discrimination learning, the peak shift, and behavioral contrast. Journal of the Experimental Analysis of Behavior, 1968, 11, 727-741.

Thomas, D. R. The role of adaptation-level theory in stimulus generalization. In G. H. Bower (Ed.), The psychology of learning and motivation (Vol. 8). New York: Academic Press, 1974.

(Received for publication September 11, 1979; revision accepted January 23,1980 .) 